\title{
Design of a Power Management Circuit for an Opto-Electro Stimulator
}

\begin{abstract}
This paper presents the design of an integrated power management circuit for use in an implantable optoelectro stimulator. It features an active rectifier with pulse width modulation (PWM) regulation to generate a $3.3 \mathrm{~V}$ regulated output, and a 3-stage high voltage charge pump that generates a $12 \mathrm{~V}$ output from a $3.3 \mathrm{~V}$ input with a $20 \mathrm{MHz}$, two-phase nonoverlapping clock generator. The circuits were designed in a $0.18-\mu \mathrm{m}$ CMOS technology requiring a chip area of $0.048 \mathrm{~mm}^{2}$. Simulation results show that the regulating rectifier has a voltage conversion efficiency of $94.3 \%$ and $92.8 \%$ with an input of $3.5 \mathrm{~V}$ and $3.6 \mathrm{~V}$, respectively. The peak power transfer efficiency for a regulated output voltage of $3.3 \mathrm{~V}$ is $\mathbf{7 0 . 7 \%}$ with an output power range of $30.3 \mathrm{~mW}$. The charge pump overall capacitance is $60 \mathrm{pF}$.
\end{abstract}

Keywords-Active rectifier, charge pump, integrated circuits, optogenetics, power management.

\section{INTRODUCTION}

Deficiencies in the peripheral nervous system can result in various neurological disorders. One example is motor neurone disease (MND), which results in a loss of the motor neurons that control the skeletal muscle activities. There are currently no therapies to delay or reverse MND, making the disease fatal [1]. Current research aims to regenerate the damaged motor nerve using stem cell-derived motor neurons. Before the muscle atrophy occurs, and the paralysis becomes irreversible, the stem cell motor neurons must be engrafted close to the targeted muscles to ensure muscle reinnervation [2]. The nerves grow at a slow rate of around $\sim 1 \mathrm{~mm} /$ day; therefore, reconnecting the motor neural circuits to the central neural system is challenging. A recent study suggests using optical stimulation to control muscle contraction after the muscles are innervated with the light-sensitive ion channel channelrhodopsin-2 (ChR2) on genetically modified, engrafted stem cell motor neurons [3]. This multidisciplinary research combines electronics, optogenetics and stem cell technologies to treat muscle paralysis caused by MND.

Implantable medical devices (IMDs) require an adequate power source. The use of batteries in implants has limitations, including a limited lifetime, requiring periodic replacement with the complications of surgery. Although rechargeable batteries are quite common in implants, their large size causes a limitation on the device miniaturization. Inductive wireless coupling has been widely used [4]-[6]. In the conventional two-step ac-dc conversion, an active rectifier followed by a dc regulator is used to convert the wirelessly transmitted ac signal to a regulated dc voltage supply. The power conversion efficiency (PCE) and voltage conversion efficiency (VCE) should be maximized to avoid reaching the specified human tissue specific absorption rate (SAR), also obtaining a high overall system efficiency [4]. An active rectifier with highspeed comparators has been proposed to boost the output voltage whenever it is low [5]. The output of such rectifiers tends to peak to the input voltage regardless of the required output voltage supply. Combining rectification and regulation into one stage can improve the overall efficiency, decrease the chip size, and better control the output voltage [6].

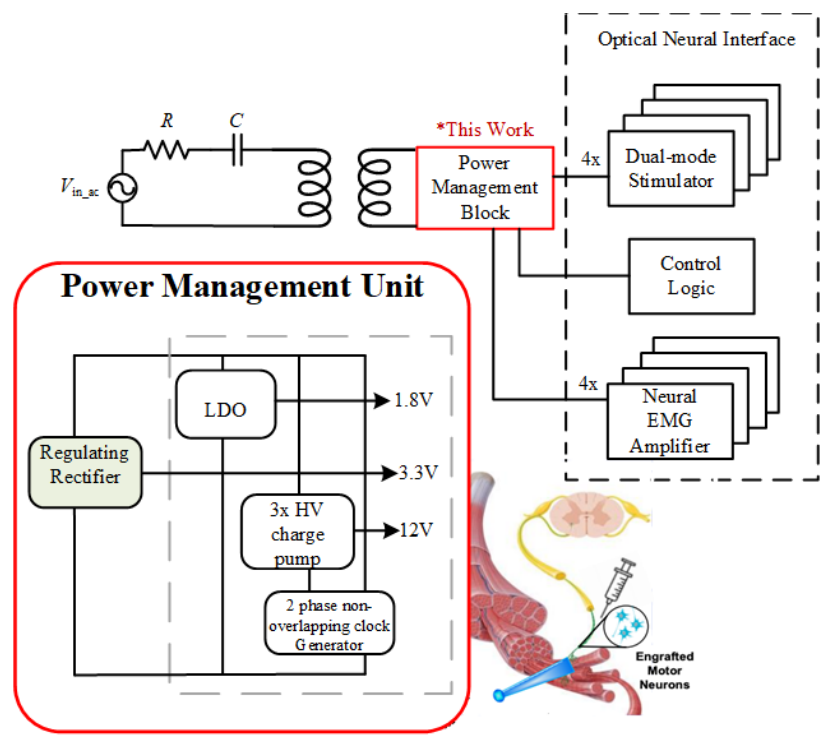

Fig. 1. Power management unit of a multi-channel opto-electro stimulator for treating motor neurone disease.

This paper focuses on the design of an active regulating rectifier, a three-stage high voltage (HV) charge pump, and the two phase non-overlapping clock generator for an optoelectro stimulator. The design also includes a dynamically body biased high speed comparator with a novel configuration of the pulse width modulation (PWM) regulation that is designed for an optical neural interface to improve the overall system efficiency and decrease the total area. The remainder of this paper is organized as follows. Section II describes the active rectifier incorporating a pulse width modulation (PWM) regulating architecture, the high-speed comparator, the design of the three-stage HV charge pump, and the design of a $20 \mathrm{MHz}$ oscillator. Section III presents the simulated performance of the power management circuit. Conclusions are drawn in Section IV.

\section{SYSTEM OVERVIEW}

The architecture of the power management circuit is shown in Fig.1. It comprises a regulating rectifier, low dropout regulator (LDO) [10], HV charge pump, and a twophase non-overlapping clock generator to generate outputs of $1.8 \mathrm{~V}, 3.3 \mathrm{~V}$ and $12 \mathrm{~V}$ to power the other implant circuits (dual mode stimulator, control logic, and neural electromyography (EMG) amplifier).

\section{A. Regulating Rectifier Architecture}

As shown in Fig. 2. the active rectifier has an input voltage of $V_{\text {in_ac }}$ which is the difference between $V_{+}$and $V_{-}$. The pair of multiplexers are connected to the gates of the two pMOS (MRP1 and $M R P 2$ ) selecting to drive either the high-speed comparator (COM1 and COM2) or the input voltage $\left(V_{+}\right.$or $V_{-}$) depending on the output of the PWM controller. The comparators (COM1 and COM2) drive the cross-coupled nMOS (MRN1 and MRN2) via the inverters. A dynamic body 


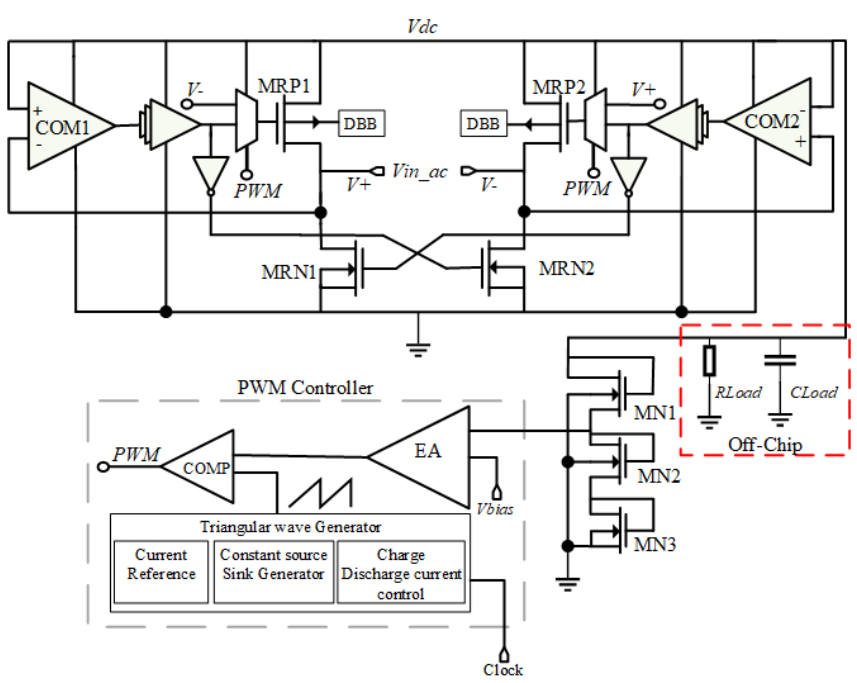

Fig. 2. Regulating rectifier with PWM Controller including the off-chip resistor and capacitor.

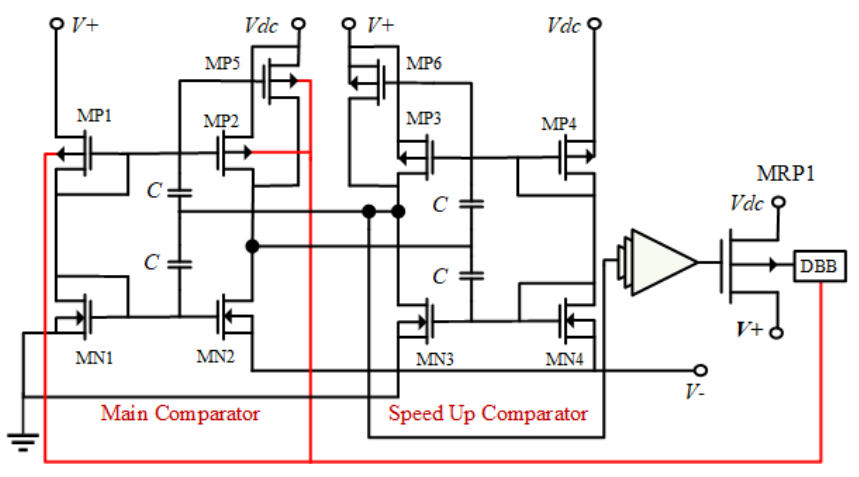

Fig. 3. Proposed high-speed comparator with body biasing technique to the pMOS of the main comparator.

biased (DBB) control technique [11] was implemented on the power transistors of the active rectifier to automatically connect to the highest potential between the input voltages $\left(V_{+}\right.$and $\left.V_{-}\right)$, and the output voltage, $\mathrm{V}_{\mathrm{dc}}$ to avoid latch up.

For regulation, a PWM controller alters the duty cycle based on the error between the set voltage $V_{\text {bias }}$ and the measured output Voltage, $V_{d c}$ that is very nearly equal to the desired value. The voltage output is connected to three diode connected transistors $(M N 1, M N 2$, and $M N 3)$ that are used for the PWM controller and also an off chip resistor load, $R_{\text {Load }}$, is added for simulations. The design also includes a $C_{\text {Load }}$ of $3 \mathrm{nF}$ off-chip filter capacitor to reduce the ripple of $V_{d c}$. Apart from maintaining the regulation, it is desirable to retain the power losses especially in implantable applications which require high efficiency. PWM can offer accurate regulation of the output voltage to keep it in the required range at the desirable range of $3.3 \mathrm{~V}$. The error amplifier $(E A)$, a comparator $(C O M P)$, and the triangle wave generator are included in the PWM Controller as shown in Fig. 2. The triangle wave generator consists of a current reference, constant source/sink current generator, and a charge discharge current control. The triangle wave generator output can be inputted into comparator (COMP) with the output of the error amplifier to generate the PWM signal. In the design, when $\mathrm{PWM}=$ Logic 0 the gate of $M R P 1$ and $M R P 2$ are connected to positive or negative rails $\left(V_{+}\right.$or $\left.V_{-}\right)$. When PWM $=$Logic 1 , they are connected to the comparators (COM1 and COM2).

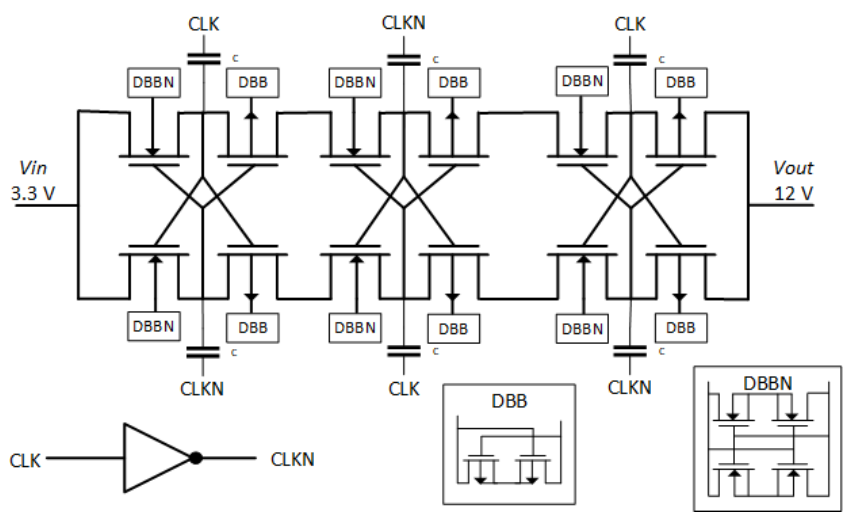

Fig. 4. Proposed latch design of three stage charge pump with body biasing of both nMOS and pMOS transistors.

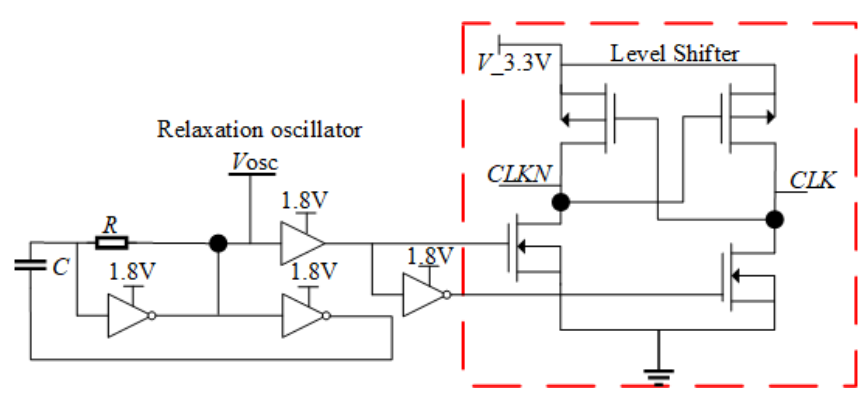

Fig. 5. Oscillator with level shifter to generate $20 \mathrm{MHz}$ CLKN and CLK.

\section{B. Proposed High Speed Comparator Design}

In Fig. 2. the comparators (COM1 and COM2) design is shown in Fig. 3. Consisting of both a main comparator and a high-speed comparator. The comparator compares the two inputs $V_{d c}$, which is the regulated voltage output, and the two rail voltage inputs $\left(V_{+}\right.$and $\left.V_{-}\right)$. The cross-coupled comparator arrangement [5] improved the rectifier's efficiency, the conduction time of the active diodes in the rectifier, and decreases the reverse leakage current. The body terminals of transistors $M P 1, M P 2$, and $M P 5$ in the main comparator are dynamically biased with the rectifier power transistor to avoid body effect and decrease the reverse current. This technique can enhance the performance of the comparators.

\section{Three Stage Charge Pump Architecture}

A widely used charge pump topology to generate boosted voltage is the Dickson charge pump with diode-connected CMOS transistors [9]. However, it suffers from a voltage drop equal to the threshold voltage in order to operate in the forward biased region. It also suffers from an increase in the threshold voltage that is caused by the body effect, which can degrade the pumping efficiency. The latch charge pump from [10] was adopted to ensure highly efficient pumping operation and to minimize the voltage drop in the charge. Existing stimulators use a large capacitor of $1 \mu \mathrm{F}$ implemented by offchip flying capacitors that are switching at a low frequency of $100 \mathrm{kHz}$, which can take up more silicon area than the stimulator itself [11]. The number of stages in the charge pump can be decided using the current consumption minimization strategy [12]. As a result, a three-stage charge pump as shown in Fig. 4 is used to boost the $3.3 \mathrm{~V}$ generated by the active regulating rectifier to produce $12 \mathrm{~V}$ output voltage. The respective body terminals of the switches are dynamically biased to make sure the substrate and n-well are 


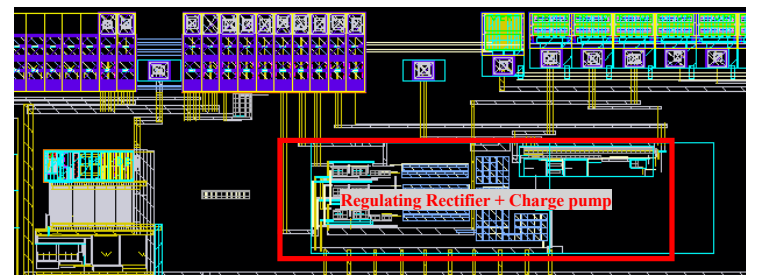

Fig. 6. The chip layout of the regulating rectifier and the charge pump.

always connected to the correct voltage during operation [11]. The implemented switches use deep n-well nMOS transistor and has two pairs of dynamic body bias to make sure that its substrate and n-well are always connected to the correct terminal. In Fig. 5, a $20 \mathrm{MHz}$ oscillator is adopted containing a relaxation oscillator feedback loop that is under $1.8 \mathrm{~V}$ [13], and a level shifter at $3.3 \mathrm{~V}$.

\section{Simulated RESUltS}

The circuit layout is shown in Fig. 6. It includes the regulating rectifier, charge pump, and the LDO within an area of $0.048 \mathrm{~mm}^{2}$.

\section{A. Regulating Rectifier}

The simulated results were carried out with a $13.56 \mathrm{MHz}$ input frequency with an output load of $R_{\text {Load }}=0.33 \mathrm{k} \Omega$ and $C_{\text {Load }}=3 \mathrm{nF}$ capacitor. Fig. 7 shows the rectified dc output $V_{d c}=3.3 \mathrm{~V}$ when $V_{\text {in_ac }}$ is $3.5 \mathrm{~V}$ and $3.6 \mathrm{~V}$. It also shows the output of the error amplifier that helps in creating the PWM controller signal for regulation. The maximum voltage conversion efficiency (VCE) obtained is around 94.3\% when the input is $3.5 \mathrm{~V}$ and $92.8 \%$ when the input is $3.6 \mathrm{~V}$. The VCE is defined as

$$
\eta_{\text {Voltage }}=\frac{V_{d c}}{\max \left(\left|V_{\text {in_ac }}\right|\right)}
$$

and PCE is defined as

$$
\eta_{\text {Power }}=\frac{P_{\text {out_dc }}}{P_{\text {in_ac }}}=\frac{P_{\text {Load }}}{P_{\text {Load }}+P_{\text {Loss,total }}}
$$

The output power is calculated by taking the average output dc power, $P_{\text {out } \_d c}$ divide it by the $P_{\text {in_ac }}$, which is the integration of the product of the differential voltage, $V_{i n_{-} a c}$, across the ac source, and the current of the source. Where $P_{\text {Load }}$ is the output power, and $P_{\text {Loss,total }}$, includes the charging and discharging power consumption, the comparators (COM1, COM2, and COMP), error amplifier, and the PWM controller. The peak measured PCE is $70.7 \%$, and it is achieved at an input dc voltage of $3.5 \mathrm{~V}$. It is crucial to optimize the size of the transistors considering the power consumption. The optimal size ratio of the pMOS and nMOS transistors proven in [14] can be found from

$$
\left(\frac{W_{p}}{W_{n}}\right)_{o p t}=\sqrt{\frac{K_{n}\left(V_{d c}-V_{T h N}\right)}{K_{p}\left(V_{d c}-\left|V_{T h P}\right|\right)}}
$$

The width of both pMOS, $W_{p}$, and nMOS, $W_{n}$, are optimized. $K_{p}$ and $K_{n}$ are the pMOS and nMOS transconductances, respectively. Having larger transistors decreases the $R_{\text {on }}$ loss, however, it increases the switching loss and comparator delays. In [15], the input frequency, $f_{\text {input }}$, is minimized to 2 $\mathrm{MHz}$ to obtain a high PCE, however, the design still suffers from a low VCE.
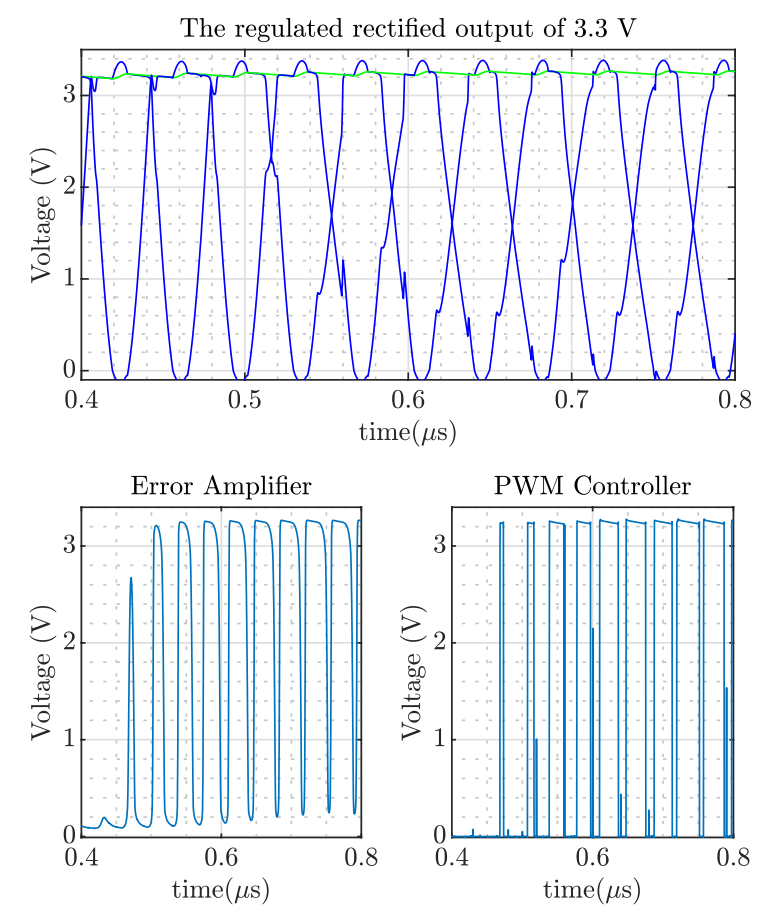

Fig. 7. The simulated results show the output of the active regulating rectifier of $3.3 \mathrm{~V}$, the output of the error amplifier, and the output of the PWM Controller.

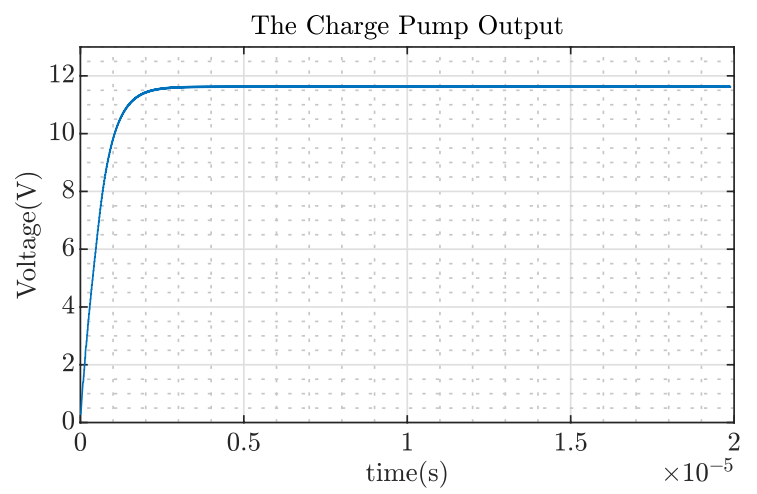

Fig. 8. Shows the output of the charge pump, which is around $11.8 \mathrm{~V}$.

\section{B. Three Stage Charge Pump}

In the charge pump design, the charge is pushed from the supply, $V_{\text {in }}$, to the output node, $V_{\text {out }}$, stage by stage. The output voltage of the charge pump circuit can be pumped to a higher voltage than the inputted voltage. The voltage fluctuation of the pumping nodes, $\Delta V$ can be expressed as

$$
\Delta V=V_{C L K}\left(\frac{C_{\text {pump }}}{C_{\text {pump }}+C_{\text {parasitic }}}\right)-\frac{I_{\text {out }}}{f\left(C_{\text {pump }}+C_{\text {parasitic }}\right)}
$$

where $V_{\text {Clock }}$ is the voltage amplitude of the clock signals used, $C_{\text {pump }}$ is the pumping capacitance, and $C_{\text {parasitic }}$ is the parasitic capacitance of each pumping stage. $I_{\text {out }}$ is the current output, and $f$ is the clock frequency. When taking $V_{\text {Clock }}$ as the same voltage level as the power supply voltage, $V_{\text {in }}$, the voltage fluctuation can be expressed as

$$
\Delta V \approx V_{\text {clock }}=V_{\text {in }}
$$

The output voltage, $V_{\text {out }}$, for the latch charge pump with charge transfer at steady state with the assumption that the vol- 
TABLE I. COMPARISON OF REGULATING RECTIFIER WITH PREVIOUS WORKS

\begin{tabular}{|c|c|c|c|c|c|}
\hline & $\begin{array}{c}\text { Lee }[14] \\
\text { TCAS-I11 }\end{array}$ & $\begin{array}{l}\mathrm{Li}[16] \\
\mathrm{JSSC} 15\end{array}$ & $\begin{array}{l}\text { Kim [6] } \\
\text { JSSC17 }\end{array}$ & $\begin{array}{c}\text { Erfani [15] } \\
\text { TBioCAS20 }\end{array}$ & This Work \\
\hline Process & $\begin{array}{l}0.5-\mu \mathrm{m} \\
3 \mathrm{M} 2 \mathrm{P} \\
\text { CMOS }\end{array}$ & $\begin{array}{c}0.35-\mu \mathrm{m} \\
\text { CMOS }\end{array}$ & $\begin{array}{c}0.18-\mu \mathrm{m} \\
1 \mathrm{P} 8 \mathrm{M} \\
\text { CMOS } \\
\text { SOI }\end{array}$ & $\begin{array}{c}0.18-\mu \mathrm{m} \\
\mathrm{TSMC} \\
1 \mathrm{P} / 6 \mathrm{M}\end{array}$ & $\begin{array}{c}0.18-\mu \mathrm{m} \\
\text { CMOS }\end{array}$ \\
\hline $\begin{array}{l}\text { Freq. } \\
(\mathrm{MHz})\end{array}$ & 13.56 & 13.56 & 144 & $1-10$ & 13.56 \\
\hline$V_{\text {in_ac }}(\mathrm{V})$ & 3.8 & NA & $0.98-1.5$ & $1.5-3.3$ & 3.5 \\
\hline$V_{d c}(\mathrm{~V})$ & 3.12 & 3.6 & 1 & $1.5-2.5$ & 3.3 \\
\hline$R_{\text {load }}(\mathrm{k} \Omega)$ & 0.5 & $0.2,1$ & 8 & 0.1 & 0.33 \\
\hline $\begin{array}{l}P_{\text {out }, \text { Max }} \\
(\mathrm{W})\end{array}$ & $20 \mathrm{~m}$ & $102 \mathrm{~m}$ & $700 \mu$ & $65 \mathrm{~m}$ & $30.3 \mathrm{~m}$ \\
\hline VCE (\%) & 82.2 & NA & 92 & 75.8 & 94.3 \\
\hline $\begin{array}{l}\text { PCE Peak } \\
(\%)\end{array}$ & 87 & 92.6 & 54 & $\begin{array}{c}90.7 \text { (@2 } \\
\text { MHz) }\end{array}$ & 70.7 \\
\hline Reg. & NA & PWM & Hybrid & $\begin{array}{c}\text { PWM + } \\
\text { PFM }\end{array}$ & PWM \\
\hline $\begin{array}{c}\text { Area } \\
\left(\mathrm{mm}^{2}\right)\end{array}$ & 0.4 & 0.18 & 0.0078 & NA & a. 0.048 \\
\hline
\end{tabular}

tage drop across the pumping switches is zero and (5) is applicable. A simply reduced equation the three stages charge pump circuit can be expressed as

$$
V_{\text {out }}=(n+1) V_{\text {in }}-V_{t}-\frac{n I_{L}}{f C_{\text {pump }}}
$$

Where $\mathrm{n}$ is the number of stages, $V_{t}$ is the threshold voltage of MOS transistors, and $I_{L}$ is the load current. Therefore, the larger the $C_{\text {pump }}$ is the larger the $V_{\text {out }}$. The $P_{\text {out }}$ of the charge pump can be calculated as

$$
P_{\text {out }}=V_{\text {out }} I_{\text {out }}=\frac{V_{\text {out }}{ }^{2}}{R_{\text {Load }}}
$$

The theoretical maximum power efficiency of the charge pump is

$$
\eta_{C P \text { Max }}=\frac{1}{n+1+\alpha\left(\frac{V_{\text {out }}}{V_{\text {in }}}\right)}-\frac{V_{\text {out }}}{V_{\text {in }}}
$$

Where $\alpha$ is multiplier factor assumed to be 0.2 [17]. The simulated results were carried out with $20 \mathrm{MHz}$ clock, $10 \mathrm{pF}$ $C_{\text {pump }}$, and $60 \mathrm{pF}$ output capacitor. The output is around 11.8 $\mathrm{V}$ with a resistor of $50 \mathrm{k} \Omega$ as shown in Fig. 8. The power conversion efficiency is $92.2 \%$. The output is restricted to be under $12 \mathrm{~V}$ to ensure that the deep n-well in the transistors is not damaged when the load is increased.

\section{CONCLUSION}

In this paper, an active rectifier operating in $13.56 \mathrm{MHz}$ with a high-speed comparator architecture is implemented. The design is implemented with PWM controller for regulating and having an output of $3.3 \mathrm{~V}$. The proposed design help reduces power dissipation. The results show the proposed active regulating rectifier achieves a peak PCE of $70.7 \%$ under a $0.33 \mathrm{k} \Omega$ load resistor and a high VCE at $94.3 \%$ under the loading resistor. The charge pump is also designed to be a three HV charge pump for the stimulator design requirements. Future work will focus on implementing an adiabatic switch control signals to enhance the performance of the charge pump and improve efficiency.

\section{REFERENCES}

D. Bäumer, K. Talbot, and M. R. Turner, "Advances in motor neurone disease," J. R. Soc. Med., vol. 107, no. 1, pp. 14-21, Jan. 2014.

S. D. Klapper et al., "On-demand optogenetic activation of human stem-cell-derived neurons," Sci. Rep., vol. 7, no. 1, Dec. 2017

Bryson JB, et al., "Optical control of muscle function by transplantation of stem cell-derived motor neurons in mice." Science, 344 (6179), 94-97, April 2014.

S. Guo and H. Lee, "An Efficiency-Enhanced CMOS Rectifier With Unbalanced-Biased Comparators for TranscutaneousPowered High-Current Implants," in IEEE Journal of Solid-State Circuits, vol. 44, no. 6, pp. 1796-1804, June 2009.

H. Cha, W. Park and M. Je, "A CMOS Rectifier With a CrossCoupled Latched Comparator for Wireless Power Transfer in Biomedical Applications," in IEEE Transactions on Circuits and Systems II: Express Briefs, vol. 59, no. 7, pp. 409-413, July 2012.

C. Kim, S. Ha, J. Park, A. Akinin, P. P. Mercier and G.

Cauwenberghs, "A 144-MHz Fully Integrated Resonant Regulating Rectifier With Hybrid Pulse Modulation for mmSized Implants," in IEEE Journal of Solid-State Circuits, vol. 52, no. 11, pp. 3043-3055, Nov. 2017.

[7] R. Jacob Baker, "CMOS Circuit Design, Layout and Simulation," IEEE Press, vol. 34, no. 2nd Edition, pp. 1-2, 2005.

[8] M. Ghovanloo and K. Najafi, "Fully integrated wideband highcurrent rectifiers for inductively powered devices," in IEEE Journal of Solid-State Circuits, vol. 39, no. 11, pp. 1976-1984, Nov. 2004.

[9] J. F. Dickson, "On-chip high-voltage generation in MNOS integrated circuits using an improved voltage multiplier technique," in IEEE Journal of Solid-State Circuits, vol. 11, no. 3, pp. 374-378, June 1976.

[10] Ming-Dou Ker, Shih-Lun Chen and Chia-Shen Tsai, "Design of charge pump circuit with consideration of gate-oxide reliability in low-voltage CMOS processes," in IEEE Journal of Solid-State Circuits, vol. 41, no. 5, pp. 1100-1107, May 2006.

[11] A. Abdi, H. S. Kim and H. Cha, "A High-Voltage Generation Charge-Pump IC Using Input Voltage Modulated Regulation for Neural Implant Devices," in IEEE Transactions on Circuits and Systems II: Express Briefs, vol. 66, no. 3, pp. 342-346, March 2019.

[12] G. Palumbo and D. Pappalardo, "Charge Pump Circuits: An Overview on Design Strategies and Topologies," in IEEE Circuits and Systems Magazine, vol. 10, no. 1, pp. 31-45, First Quarter 2010.

[13] V. W. Leung et al., "A CMOS Distributed Sensor System for High-Density Wireless Neural Implants for Brain-Machine Interfaces," ESSCIRC 2018 - IEEE 44th Eur. Solid State Circuits Conf., pp. 271-273, 2018.

[14] H. -M. Lee and M. Ghovanloo, "An Integrated Power-Efficient Active Rectifier With Offset-Controlled High Speed Comparators for Inductively Powered Applications," in IEEE Transactions on Circuits and Systems I: Regular Papers, vol. 58, no. 8, pp. 17491760, Aug. 2011

[15] R. Erfani, F. Marefat and P. Mohseni, "A Dual-Output SingleStage Regulating Rectifier With PWM and Dual-Mode PFM Control for Wireless Powering of Biomedical Implants," in IEEE Transactions on Biomedical Circuits and Systems, vol. 14, no. 6, pp. 1195-1206, Dec. 2020.

[16] X. Li, C. Tsui and W. Ki, "A 13.56 MHz Wireless Power Transfer System With Reconfigurable Resonant Regulating Rectifier and Wireless Power Control for Implantable Medical Devices," in IEEE Journal of Solid-State Circuits, vol. 50, no. 4, pp. 978-989, April 2015.

[17] G. Palumbo, D. Pappalardo and M. Gaibotti, "Charge-pump circuits: power-consumption optimization," in IEEE Transactions on Circuits and Systems I: Fundamental Theory and Applications, vol. 49, no. 11, pp. 1535-1542, Nov. 2002. 